\title{
Mobile Assessment Tools
}

\author{
http://dx.doi.org/10.3991/ijes.v2i3.3816 \\ K. Henke, K. Debes, H.-D. Wuttke, A. Katzmann \\ Technische Universität Ilmenau, Thüringen, Germany
}

\begin{abstract}
This paper describes the concept for a Webbased, database supported e-Learning Test, Examination and Assessment System, called TEASE, that can be used via the Internet and is therefore suitable for both local and remote examination preparation as well as for examination within lab courses (entry test) or during lectures (big online exams in lecture halls). Another topic of this contribution is the improvement of the mandatory authentication for a multitude of students during written examinations in large lecture halls - the usage of barcodes to register both the student as well as the issued exercise sheet of the exam.
\end{abstract}

Index Terms-Assessment Systems, Blended Learning, Interactive Learning Environments, M-Learning, Web-based Education.

\section{INTRODUCTION}

The increasing capacity of wireless communication and the growing number of mobile devices (e.g. smartphones and tablets) on the one hand as well as modern Internet technologies like JavaScript, HTML5 and Web Sockets on the other hand provide new possibilities and challenges in the area of mobile learning (M-Learning) $[1,2]$.

In our contribution we would like to present a concept for a Web-based, database supported e-Learning Test, Examination and Assessment System, called TEASE [3], that can be used via Internet and is therefore suitable for both local and remote examination preparation as well as for examination within lab courses (entry test) or during lectures (big online exams in lecture halls). This solution is also intended for the use in learning modules and remote laboratories dealing with the design of complex digital control systems within the newly established Tempus project "ICo-op - Industrial Cooperation and Creative Engineering Education based on Remote Engineering and Virtual Instrumentation", founded by the European Commission within the program "Tempus", Grant No 530278TEMPUS-1-2012-1-DE-TEMPUS-JPHES [4].

During the development of the TEASE system, priority was given to platform independence. Nowadays the usage of smartphones and tablets is common in everyday life. Students can access and share information, communicate, collaborate and learn anywhere at any time. This means that the described TEASE system should be usable independent of user and system properties from any place at any time. Beyond that there should be the possibility to integrate this system into Learning Management Systems (LMS). At various universities diverse LMS have been established in the last years. While planning the TEASE system, we choose not to implement mechanisms for a special LMS. Therefore a realization as HTML5 RIA (Rich Internet Application - a consistent enhancement of previous Java applets) was chosen as common platform, offering the chance to use the system stand-alone without Internet connectivity as well.

TEASE is generally made to be used for examinations (e.g. entry test for online labs or big online exams in lecture halls). Furthermore it should allow exercising learning content anonymously (which means without any login procedure and grading). Tasks and examinations should be managed by database support. An assessment happens either by displaying the correct results (during exercise mode) or by calculating a mark (during examination mode).

Figure 1 gives an impression of the mobile usage of the $T E A S E$ system in Exercise Mode on an iPad.

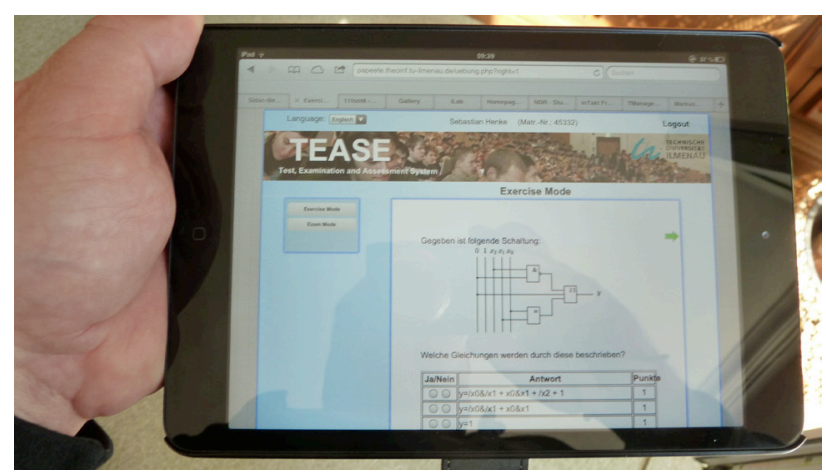

Figure 1. Using the TEASE system on an iPad

In the following we will discuss the main components of our Web-based and database supported M-Learning Test, Examination and Assessment System as well as possible working modes of the system (offline test, online test and examination) by examples. This system is based on the concepts of the Java-based $A W i P^{l}$ system, developed at the Department of Integrated Communication System at the Ilmenau University of Technology. For a detailed description of the $A W i P$ system see $[5,6,7]$.

\section{THE TEASE SYSTEM}

\section{A. Requirements}

During the development of the Test, Examination and Assessment System TEASE priority was given to platform independence. The system should be usable from any place at any time by different users with different system properties - especially by using mobile devices in the area of mobile learning. Beyond that there should be the possibility to integrate this system into Learning Management Systems (LMS). At various universities there have been established diverse LMS. While planning the TEASE system we choose not to implement mechanisms for a special

\footnotetext{
${ }^{1}$ AWiP stands for „Applet zur Wissensüberprüfung im Praktikum“ - in
} English: an applet for knowledge testing in laboratory courses. 
LMS. Therefore a realization as HTML5 RIA (Rich Internet Application - a consistent enhancement of previous Java applets) was selected as common platform offering the chance to use the system stand-alone without Internet connectivity in addition.

During the implementation of the Test, Examination and Assessment System great importance was attached to the

- reuse of the applied methods and tools for similar problems,

- reuse of the developed software modules,

what finally leads to synergy effects in the production process of different test and assessment tools.

\section{B. Architecture}

The TEASE system essentially consists of the following components (see Figure 2):

(1) the test system TEASE itself (TEASE core),

(2) a databased supported Collection of Tasks (TEASE DB),

(3) the TEASE Player and

(4) an Administration Tool.

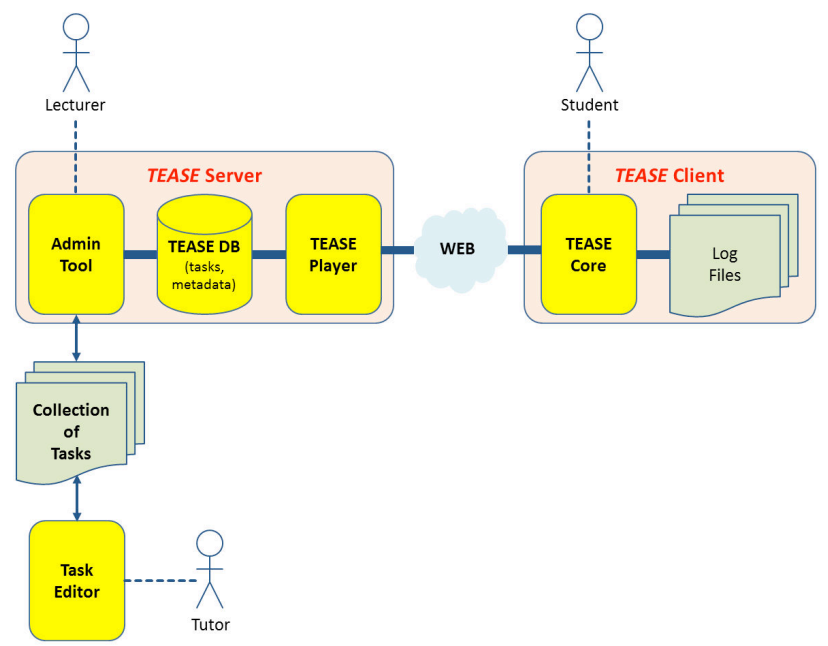

Figure 2. Components of the TEASE system

The complete system is implemented multilingual. All text messages and labels are stored in the TEASE DB and can be exchanged during run time.

\section{(1) TEASE core}

The user interface on the client site is realized as HTML5 RIA and provides tasks from a (randomly selectable) text file and performs an evaluation of the student's result. The student can solve his tasks in any sequence and is able to interrupt his work for a certain time. ed:

At present the following types of tasks are implement-

- Multiple Choice Tasks (see Figure 3),

- Cloze Tasks (see Figure 4),

- RIA Tasks - will be supported as soon as the former Java applets are realized in RIA architecture as well (see [7]).

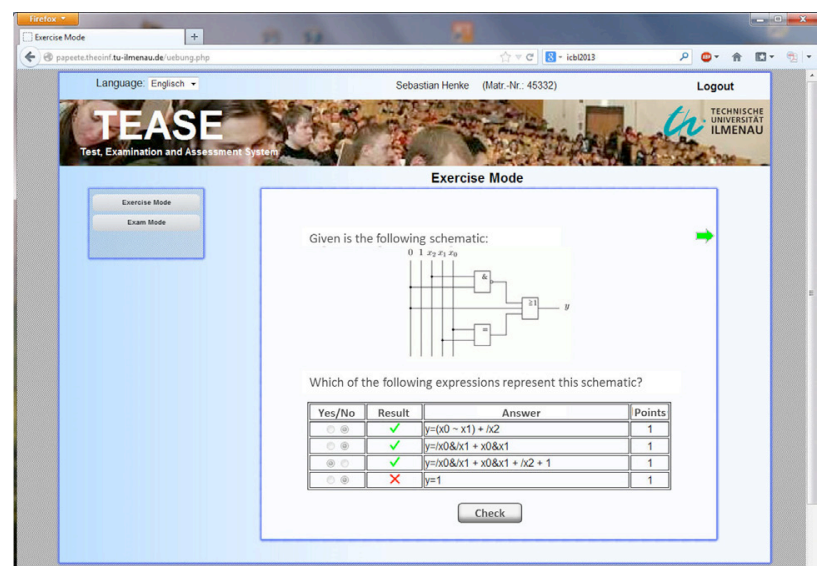

Figure 3. TEASE example for Multiple Choice (with check)

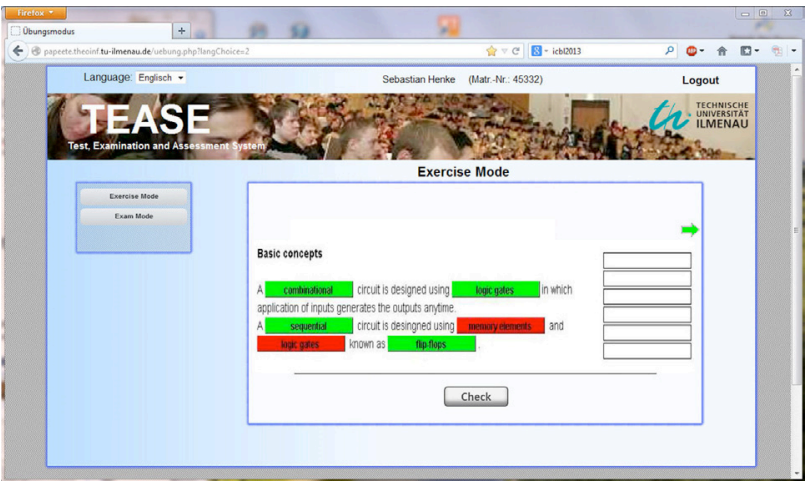

Figure 4. TEASE example for Cloze Tasks (with check)

\section{(2) Databased Collection of Tasks}

The core is an interpreter module for the segments of the Task collection. As shown in Figure 5, all task segments consist of a label to specify the type followed by the content (text, image, applet etc.).

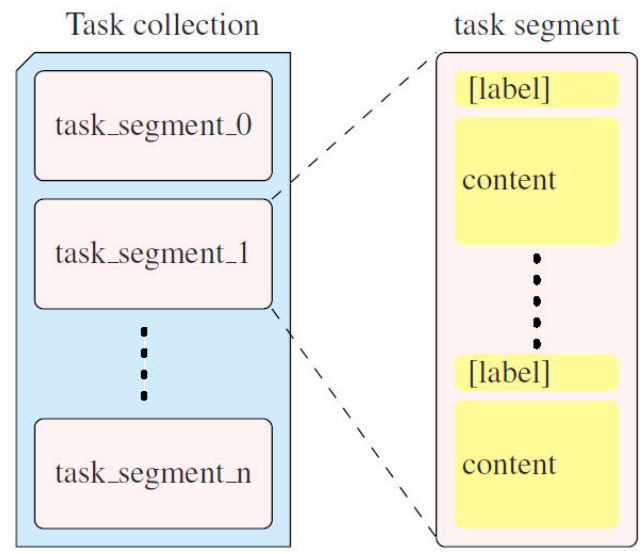

Figure 5. Structure of a Collection of Tasks

All tasks follow a simple syntax and are stored centrally in the TEASE DB. Furthermore it is possible to use a locally stored database in which text file based tasks are easily importable (see section Administration Tool), which allows editing those by any editor (offline as well). Required attributes and metadata were determined by performing an analysis of demands and use cases [8].

Another feature is the possibility to modify the system for different requirements (e. g. different courses of study, lectures and labs) without any change of code. 
For the administration of all data the database MySQL 5.6 was chosen.

\section{(3) TEASE Player}

For a universal configuration of the Test, Examination and Assessment System the TEASE system itself was enhanced by the TEASE Player. This module is responsible for the automated task generation - depending on the chosen metadata (e.g. main topics, difficulty, number of points, coding of marks, time limit, ...) for the actual assessment activity (see also section $\mathrm{C}$ ).

Within the Exercise mode the student can generate multiple tasks suited for his requirements.

For an Examination, the lecturer or tutor can create a collection of tasks by using corresponding metadata.

\section{(4) Administration Tool}

For an effective handling of the whole TEASE system an administration tool is necessary for the preparation of tasks, examinations and metadata. It offers the following features:

- to create and update tasks using a build-in or external editor,

- to import and export tasks,

- to create collections of task for examinations,

- to define metadata for tasks, examinations and libraries of examinations,

- to define time slots and exam passwords for a safe usage of the system during examinations.

\section{Supported working modes}

The following working modes are supported at the present time:

\section{- Offline Test}

Here the student can generate a collection of tasks suited for his requirements through the TEASE Player by using metadata. Later he is able to solve the given tasks and get an assessment of the results without Web support by using only the TEASE stand-alone system.

This mode will be only available for PC based systems. It is not available for mobile devices and that's why not the focus of this article.

\section{- Online Exercise Mode}

In this case the student is given a number of tasks (either randomly or suited for his requirements) by the TEASE Player - running on the TEASE Server site, that he can process online and get an assessment by the TEASE system corresponding to his answers.

Within the exercise mode the student can generate multiple tasks suited for his requirements. It means, he can select

- tasks for a special lecture,

- tasks for a special topic (independent of special lectures),

- tasks for a special topic of a specific lecture,
- open and not answered tasks if he interrupts the examination session for a certain time. In this case the actual processing status is saved in a log file within in the TEASE DB.

\section{- Examination}

During an examination a log dataset will be generated automatically in the background which will record the actual state of the student's work. That's why a login procedure is required at the beginning of the examination. At the Ilmenau University of Technology the common LDAP system is used for user authentication. After that the student is given his collection of tasks to be processed in a given time in any order. When all tasks are solved (or the time limit is over) the resulting mark will be calculated (via a comparison with an existing list of marks) and displayed. As a proof the student will get a certificate with relevant data (course, name, student ID, mark, date). Furthermore, the result is accessible for examiners and supervisors via the Administration Tool.

For big online exams in lecture halls the examination mode will be activated only for a certain time slot and must be activated by an additional password (given by the supervisor) as well.

While the TEASE system is in examination mode, it is not possible to switch to any exercise mode. Furthermore it can only be closed by the supervisor using his admin password. During this closing procedure the created log file will be removed. If there is an unexpected break (without admin password) the exact state before the termination will be restored during a TEASE restart.

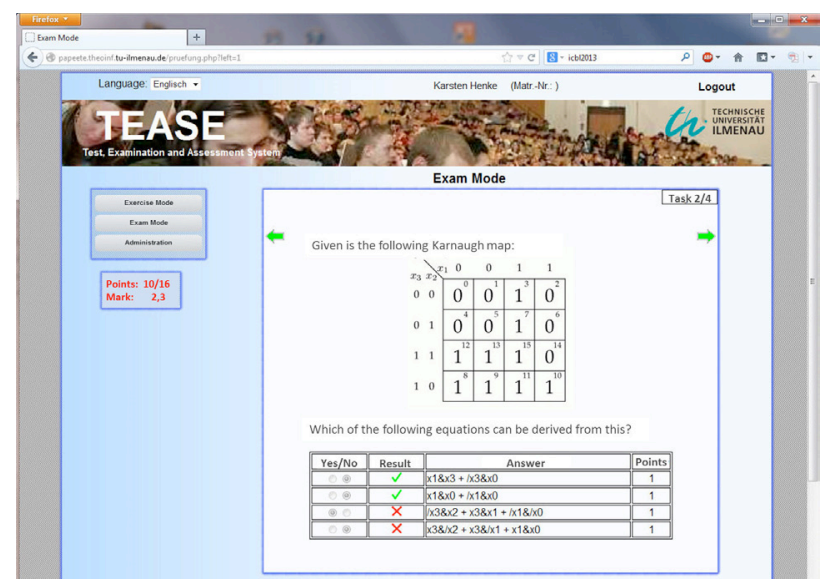

Figure 6. TEASE in Examination Mode (with scores and resulting mark)

\section{MOBILE STUdent AuthentiCATION}

Another topic of our contribution is the improvement of the mandatory authentication for a multitude of students during written examinations in large lecture halls. It is obligatory that each student has to be registered by his identification card as well as by his signature. Especially in common undergraduate courses at the Ilmenau University of Technology for different study programs the Professor/teacher will get up to 10 different attendance lists from different examination offices (from different facul- 
ties) for the authentication procedure. Especially the collection (and registration) of all written exams after completion in a certain time is an almost unsolvable problem for the attending staff. That is why we have thought about how this process can be improved.

One suitable solution is the usage of barcodes to register both the student as well as the issued exercise sheet of the exam, which will be described in the following [9].

\section{A. Requirements}

On Universities exist several ID-mechanisms and devices which can be used for authentication: On the one hand, every student has a personal student ID card with passport photograph and a unique barcode [10]. On the other hand, the university has some barcode scanners running on a suited mobile operating system [11, 12]. On these systems own additional applications can be installed (see Figure 7).
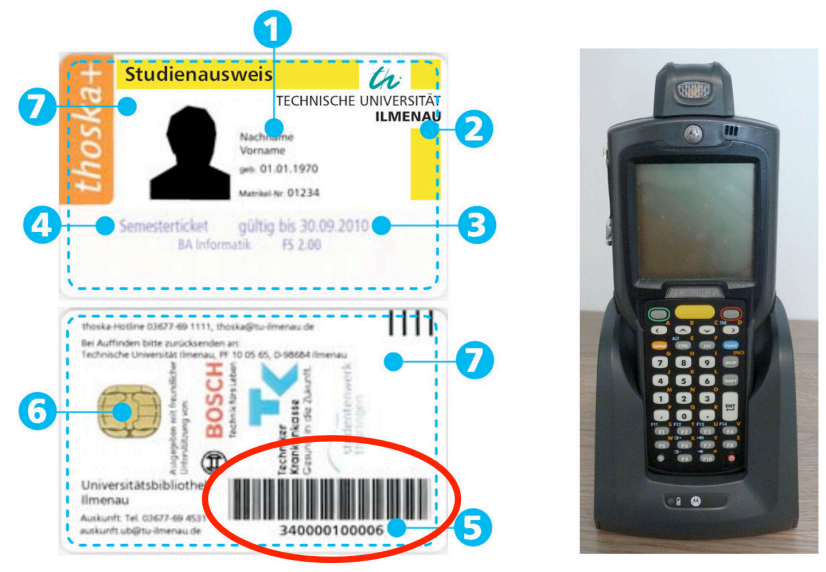

Figure 7. Thoska student ID card with barcode (5) and barcode scanner

And finally it is possible to provide each examination exercise sheet with a specific scan label, as shown in Figure 8.

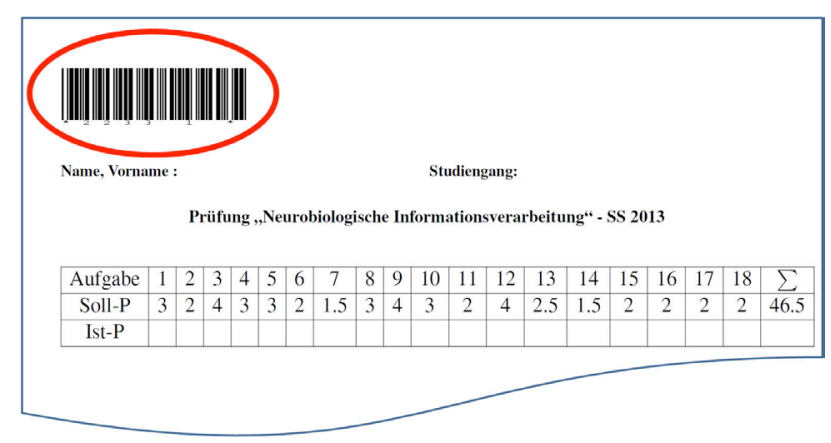

Figure 8. Example for an exercise sheet with barcode label

\section{B. Barcode generation for examinations}

A barcode label on an examination exercise sheet should contain at least

- the examination ID, defined in the university calendar as well as

- a sequential number.

At the Ilmenau University of Technology the barcode specifications Code39 and Code128 are widespread. Both can be used for alphanumeric or numeric-only barcodes.
On the Thoska ID card the Code39 is implemented. Therefor this code was chosen for this application.

To place the barcode label on each exercise sheet of the examination, there exist the following possibilities:

- The simplest way is to print out self-adhesive scan code labels (Herma, Zweckform) in the secretariat and place it manually on each copied exam - like stamps.

- The second way is to generate the scan code automatically with LaTeX or Word together with the exercises. But in this case it is necessary to print out all the exams individually - it is not possible to copy them.

For first field studies the barcode we used the LaTeX based version (see Figure 8).

\section{Student authentication during examination}

The new barcode-based examination authentication process for students will mainly take place in four steps.

\section{(1) Preparation phase}

During the preparation phase (see Figure 9) all the attendance lists for certain study courses from the faculty examination offices will be downloaded to the supervisor's notebook (a). These lists will be converted to a scanner-readable $c s v$ format and will be transferred to the barcode scanner (b). A printed copy of the attendance lists as backup is recommended (c).

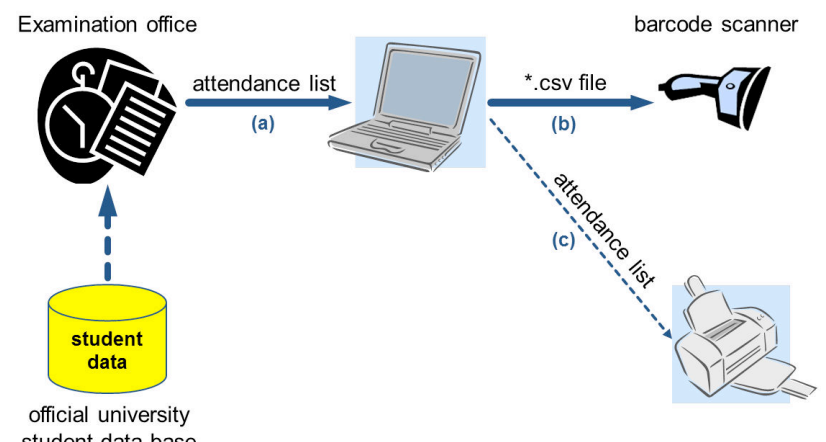

Figure 9. Phase 1: Preparation phase

\section{(2) Examination registration phase}

During the examination registration phase in the lecture hall (see Figure 9) the bar code on the student ID card as well as the bar code on each exercise sheet will be captured (a). If a student is not registered in the system yet (b), he can be entered manually (if needed) into the bar code scanner. 


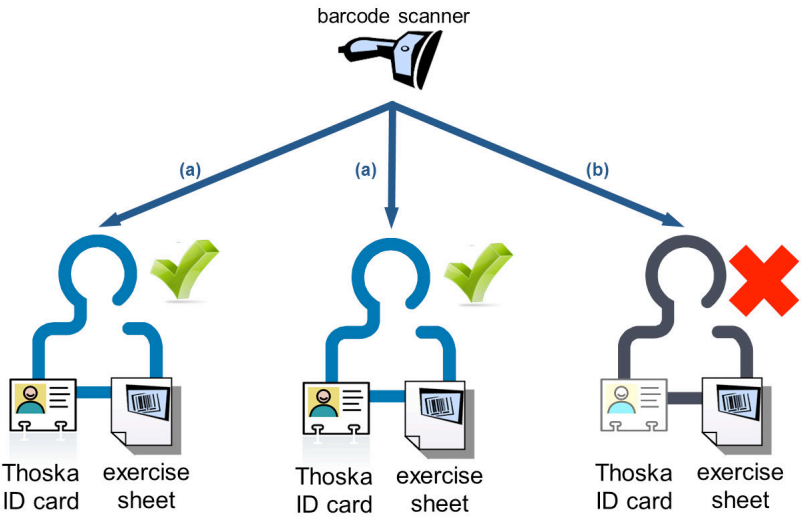

Figure 10. Examination registration phase in the lecture hall

Figure 11 gives an impression of the registration process in the lecture hall during an examination.

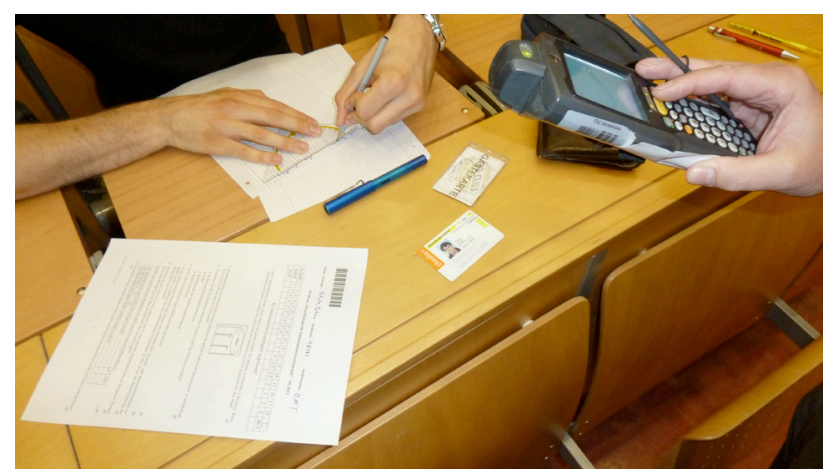

Figure 11. Impression of the examination registration phase

\section{(3) Examination collection phase}

After completion of the examination, during the exam collection phase (see Figure 12), the bar code on the exercise will confirm the handover of the exercise sheet (a). The teacher therefor receives an actual overview about missing exercise sheets as well (b).

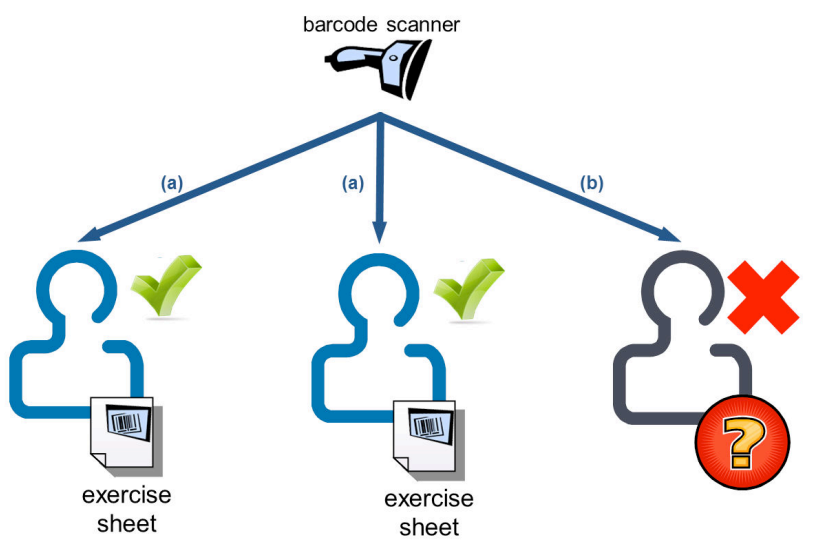

Figure 12. Examination collection phase in the lecture hall

\section{(4) Post-processing phase}

Finally, during the post-processing phase, the teacher has to upload the scanned data from the scanner to his laptop (a), print out (b) and sign the attendance lists (c) and send them back as official document to the appropriate examination offices.

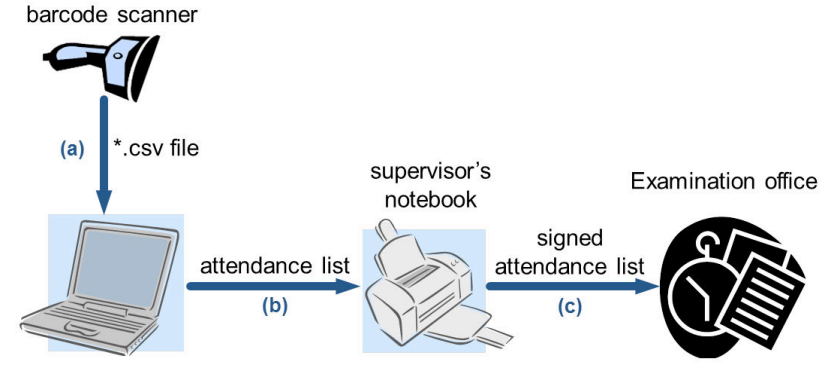

Figure 13. Phase 4: Post-processing

\section{First results}

Since the examination contains only the barcode label, it is anonymized, which allows a neutral evaluation. The system has been tested during the last three semesters with around 1000 students. The expedition of time for the authentication process as well as for the exam collection phase will be reduced to around one quarter. Furthermore, the unique assignment "exercise sheet to student" complicates fraud attempts and supports the correctness of the exercise process as well.

After initial skepticism by the Student Council, the acceptance by the students is very positive. The authentication process is very fast which finally means less disturbance and distraction during the examination time in the lecture hall. The teachers, who have so far used the system are very convinced of the solution and confirm the significant raising efficiency - combined with improved accuracy and data integrity of the overall examination process.

\section{CONCLUSION}

A universal Web-based test, examination and assessment system - called TEASE - has been discussed. The next step should be the implementation of the TEASE system into Learning Management Systems (e. g. moodle LMS). This means that students can use all the functionalities of the LMS as well as the various assessment modes of the TEASE system.

$T E A S E$ was designed especially for mobile learning:

- to be used for examination preparation (test) as well as for examinations within lab courses (e.g. as entry test) or big online exams in lecture halls,

- including the ability for both local and Web-based remote access or

- to be integrated into different Learning Management Systems (e. g. Moodle at the TU Ilmenau);

- to focus on automated task generation and assessment to gain information about student's preknowledge and knowledge acquisition in the learning process,

- to deliver information for adapting course activities and learning content (in case of adaptive LMS) and

- to provide feedback about the actual state of knowledge to students and lecturers as well.

Within the mentioned Tempus project "ICo-op" it is increasingly necessary to allow and organize a shared use of remote lab equipment. Therefore, main focus is the Web-wide usage of the discussed TEASE system. 
PAPER

Mobile AsSESSMENT TOOLS

The second topic of this article discussed the improvement of the mandatory authentication for a multitude of students during written examinations in large lecture halls using barcode labeled exercise sheets and barcode scanner. The pilot phase during the last three semesters with around 1000 students shows that it leads to a significant simplification of the authentication process during huge examinations.

\section{ACKNOWLEDGMENT}

The authors would like to thank Ronny Stricker for the inspiring discussions during the conception of the barcode authentication project.

Parts of this work are supported by the project "ICo-op - Industrial Cooperation and Creative Engineering Education based on Remote Engineering and Virtual Instrumentation" by the European Commission within the program "Tempus", Grant No 530278-TEMPUS-1-2012-1-DETEMPUS-JPHES [4].

\section{REFERENCES}

[1] Y.A. Rahim, A.N. Che Pee, M. A. Othman, "Observation on the uses of Mobile Phones to Support Informal Learning", International Journal of Interactive Mobile Technologies, Vol. 6, No 4 (2012), pp.43-45.

[2] M. György, "New Learning Spaces? M-learning's, in Particular the iPad's Potentials in Education", International Journal of Interactive Mobile Technologies, Vol. 7, No 1 (2013), pp.56-60.

[3] TEASE Website: http://papeete.theoinf.tu-ilmenau.de

[4] ICo-op project Website: http://www.ICo-op.eu

[5] K. Henke, H.-D. Wuttke, "Generator for Pre and Post Tests", Remote Engineering and Virtual Instrumentation, 29/30. June 2006, Maribor, Slovenia, Kassel University Press, ISBN 3-89958194-6, 10 pages.

[6] N. Wagner, "Computer aided test", Master's Thesis, Ilmenau University of Technology, 2007.

[7] K. Henke, "Web-based Test, Examination and Assessment System", in journal: Advanced Technology for Learning, Vol.4, Issue 3, 2007, ACTA Press, Calgary, Anaheim, Zürich, ISSN 17102251 , October 2007.
[8] N. Ludwig, "Learning environments for remote labs - concept and realization", Master's Thesis, Ilmenau University of Technology, 2005.

[9] K. Debes, "Klausurerfassung mittels Barcode" (in German), Case Study, Ilmenau University of Technology, unpublished, 2012.

[10] Thoska Homepage: http://www.uni-jena.de/thoska.html

[11] H.E. Burke, "Automating Management Information Systems: Barcode Engineering and Implementation", Thomson Learning, 1990 .

[12] E. F.Brigham, “The Barcode Manual”, Cengage Learning, 1995.

\section{AUTHORS}

Karsten Henke is with the Ilmenau University of Technology, Faculty of Computer Science and Automation, Integrated Communication Systems Group, 98684 Ilmenau, Germany, POB $10 \quad 05 \quad 65$ (e-mail: karsten.henke@tu-ilmenau.de).

Klaus Debes is with the Ilmenau University of Technology, Faculty of Computer Science and Automation, Neuroinformatics and Cognitive Robotics Lab, 98684 Ilmenau, Germany, POB $10 \quad 0565$ (e-mail: klaus.debes@tu-ilmenau.de).

Heinz-Dietrich Wuttke is with the Ilmenau University of Technology, Faculty of Computer Science and Automation, Integrated Communication Systems Group, 98684 Ilmenau, Germany, POB 100565 (e-mail: dieter.wuttke@tu-ilmenau.de).

Alexander Katzmann is with the Ilmenau University of Technology, Master Student in Computer Science at the Faculty of Computer Science and Automation, 98684 Ilmenau, Germany, POB 1005 65. He obtained his BSc. in Computer Engineering in 2013 (e-mail: alexander.katzmann@tu-ilmenau.de).

This work was supported in part by the European Commission within the program "Tempus", Grant No 530278-TEMPUS-1-2012-1-DETEMPUS-JPHES. It is an extended and modified version of a paper presented at the ICBL2013 International Conference on Interactive Computer aided Blended Learning, held 6 - 8 November 2013, in Florianópolis, Brasil. Submitted, 29 April 2014. Published as resubmitted by the authors 07 August 2014. 